\title{
Atrial fibrosis and decreased connexin 43 in rat hearts after exposure to high-intensity infrasound
}

\author{
Ana Lousinhaa ${ }^{\mathrm{a}, *}$, Gonçalo Pereira ${ }^{\mathrm{a}}$, Gonçalo Borrecho ${ }^{\mathrm{a}}$, José Brito ${ }^{\mathrm{a}}$, \\ António Oliveira de Carvalho ${ }^{\mathrm{c}}$, Diamantino Freitas ${ }^{\mathrm{c}}$, Pedro Oliveira ${ }^{\mathrm{a}}$, Maria João R. Oliveira ${ }^{\mathrm{b}}$, \\ Eduardo Antunes ${ }^{\mathrm{a}}$ \\ ${ }^{a}$ Center for Interdisciplinary Research Egas Moniz (CIIEM), Health Sciences Institute, 2829-511 Monte de Caparica, Portugal \\ ${ }^{\mathrm{b}}$ Department of Anatomy and UMIB of Abel Salazar Biomedical Sciences Institute, University of Porto, Porto, Portugal \\ ${ }^{\mathrm{c}}$ Engineering Faculty, Polo da Asprela, University of Porto, 4200-465 Porto, Portugal
}

\section{A R T I C L E I N F O}

\section{Keywords:}

Infrasound

Atrial fibrosis

Connexin 43

\begin{abstract}
A B S T R A C T
Background: Noise is an important environmental risk factor. Industrial environments are rich in high-intensity infrasound (hi-IFS), which we have found to induce myocardial and coronary perivascular fibrosis in rats. The effects of exposure to IFS on the ventricles have been studied, but not on the atria. We hypothesized that rats exposed to hi-IFS develop atrial remodeling involving fibrosis and connexin 43, which we sought to evaluate. Material and methods: Seventy-two Wistar rats, half exposed to hi-IFS (120 dB, <20 Hz) during a maximum period of 12 weeks and half age-matched controls, were studied. Atrial fibrosis was analyzed by Chromotropeaniline blue staining. The immunohistochemical evaluation of $\mathrm{Cx} 43$ was performed using the polyclonal antibody connexin-43 m diluted 1:1000 at $4{ }^{\circ} \mathrm{C}$ overnight. Digitized images were obtained with an optical microscope using $400 \times$ magnifications. The measurements were performed using image $J$ software. A two-way ANOVA model was used to compare the groups.

Results: The mean values of the ratio "atrial fibrosis / cardiomyocytes" increased to a maximum of $0.1095 \pm 0,04$ and $0.5408 \pm 0,01$, and of the ratio "CX43/ cardiomyocytes" decreased to $0.0834 \pm 0,03$ and $0.0966 \pm 0,03$, respectively in IFS-exposed rats and controls. IFS-exposed rats exhibited a significantly higher ratio of fibrosis $(p<.001)$ and lower ratio of $\mathrm{Cx} 43(p=.009)$.

Conclusion: High-intensity infrasound exposure leads to an increase in atrial interstitial fibrosis and a decrease in connexin 43 in rat hearts. This finding reinforces the need for further experimental and clinical studies concerning the effects of exposure to infrasound.
\end{abstract}

\section{Introduction}

Noise is an important environmental and occupational risk factor and it is consensual that human exposure to this aggressor can induce systemic damage, thus having an impact on public health. The characteristics of the noise stimulus that may be responsible for inducing this aggression are not fully known. The World Health Organization (WHO) Regional Office for Europe has been particularly concerned with sound pressure level $(\mathrm{dB})$ limits but also acknowledges that low frequency noise (LFN), below $200 \mathrm{~Hz}$, represents an environmental problem (WHO, Regional Office for Europe, 2011; WHO, Regional Office for Europe, 2018).

Research on the impact of LFN, below $200 \mathrm{~Hz}$, and Infrasound (IFS), below $20 \mathrm{~Hz}$, established that they are hazardous for the human body, particularly for pressure levels above 120 dB (Leventhall, 2004; Leventhall, 2007). From a physics standpoint, noise and sound are the same, defined as a pressure disturbance that propagates through a material at a speed which is dependent on the material (Beranek, 2006). When considering sound propagation, an obstacle in the sound path will cause its reflection, absorption or transmission through the object. In general, these three processes depend on the wavelength of the sound. Low frequency noises, by having longer wavelengths, are likely more transmitted through the body wall, thus affecting internal systems and organs (Leventhall, 2004; Leventhall, 2007; Beranek, 2006; Ziaran, 2014). Furthermore, high intensity-LFN and IFS can induce resonance responses in body cavities (Leventhall, 2007). The overall

\footnotetext{
* Corresponding author.

E-mail addresses: alousinha@gmail.com (A. Lousinha), carvalho@fe.up.pt (A. Oliveira de Carvalho), dfreitas@fe.up.pt (D. Freitas), mjoliveira@icbas.up.pt (M.J. R. Oliveira).
} 

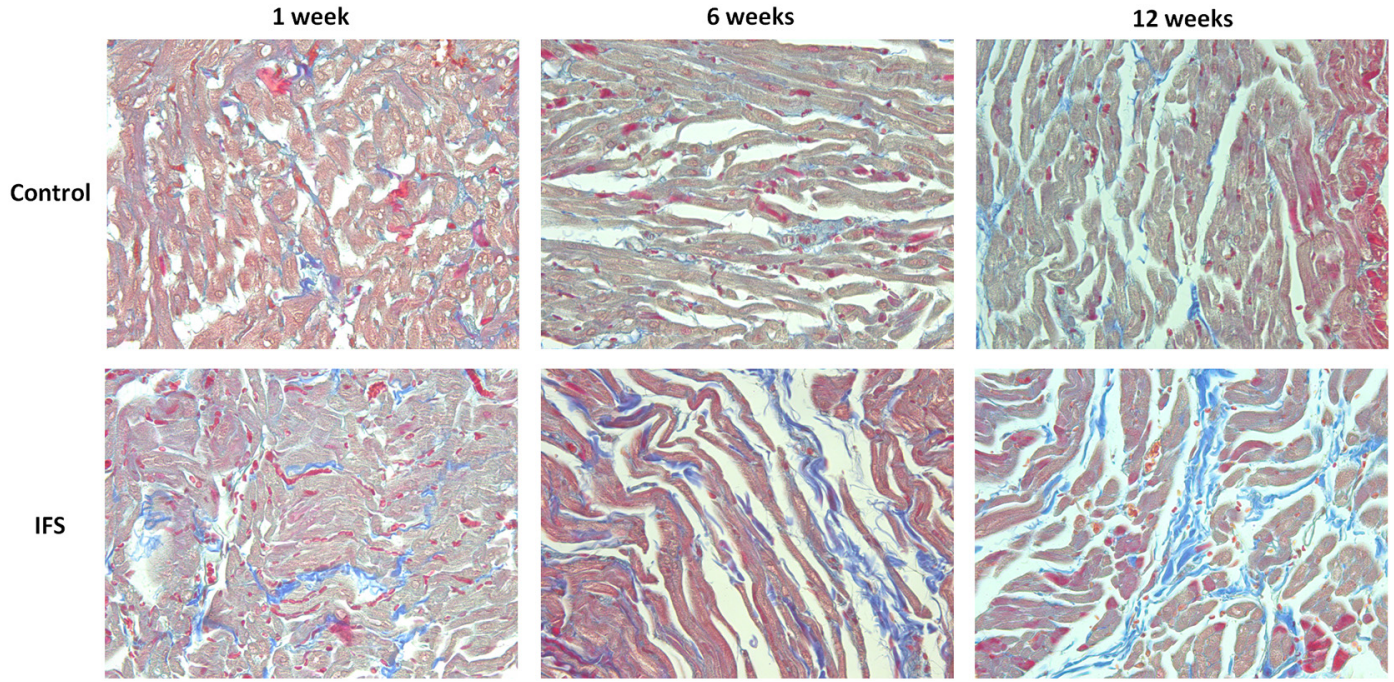

Fig. 1. Atrial interstitial fibrosis after high-intensity IFS $(120 \mathrm{~dB},<20 \mathrm{~Hz})$ exposure. Examples of CAB-stained atrial sections from control and IFS-exposed rats at 1,6 and 12 weeks [CAB, $400 \times$ ]. Note de prominent interstitial fibrosis in IFS-exposed animals. IFS - Infrasound. CAB - Chromotrope Aniline Blue. (For interpretation of the references to color in this figure legend, the reader is referred to the web version of this article.)

range of human body resonant frequencies was found to be from 2 to $16 \mathrm{~Hz}$ (Randall et al., 1997), which is almost the exact range of IFS. The displacement between organ and skeletal structures places biodynamic strain on the involved body tissue and it is known to reach its maximum under exposure to noise close to the body's resonant frequency.

Noise affects both auditory and non-auditory systems of humans exposed to it (Basner et al., 2014). The type of exposure (continuous, occasional and occupational) and its duration may trigger different responses (Ising and Kruppa, 2004; Schust, 2004). According to experimental and epidemiological studies, the non-auditory effects of noise include annoyance, sleep disturbance and psychological stress and affect the cardiovascular system (Leventhall, 2004; Leventhall, 2007; Basner et al., 2014). Other animals also possess inherent specific sound frequencies in certain tissues and organs (Pei et al., 2009). Studies on the morphological and biological effects of exposure to different types of noise, from industrial to LFN, in animal models, showed an increased volume of connective tissue and collagen fibers in different tissues and organs (Oliveira et al., 2005; Oliveira et al., 2012; Oliveira et al., 2013). In the cardiovascular system of rodents, we reported the development of perivascular fibrosis around the coronary arteries of rats exposed to industrial noise (Antunes et al., 2013a; Lousinha et al., 2015) and documented a significant fibrotic development in ventricular myocardium among rats submitted to LFN (Antunes et al., 2013b; Antunes et al., 2013c), together with a possible ventricular gap junction remodeling (Antunes et al., 2013d). We also found that IFS exposure induces coronary perivascular fibrosis (Lousinha et al., 2018).

The purpose of our study was to test the hypothesis that different periods of high intensity infrasound exposure $(120 \mathrm{~dB},<20 \mathrm{~Hz})$ can cause development of atrial interstitial fibrosis and modifications of $\mathrm{Cx} 43$ in rats, by performing a histological and immunohistochemical evaluation.

\section{Material and methods}

\subsection{Animals}

Following the 3Rs principles (Tannenbaum and Bennett, 2015), this study shares data and resources with a larger study of the effects of infrasound exposure on the pancreatic morphology and function, which was approved by the Animal Welfare Body (ORBEA) of Abel Salazar Biomedical Sciences Institute, University of Porto (Portugal), under the protocol $n^{\circ}$ 204/2017. Thus, seventy-two male Wistar rats purchased from a Spanish breeder (Charles River Laboratories España, S. A., Spain), aged 16 weeks, weighing $375.95 \pm 18.29 \mathrm{~g}$, were selected from the original sample. The postmortem collection of hearts did not alter the approved primary protocol procedures in any way. All the handling and care of the experimental animals was performed by authorized researchers (accredited by the Federation of European Laboratory Animal Science Associations, Category C) and was done in accordance with the EU Commission on Animal Protection for Experimental and Scientific Purposes (2010/63/EU) and with the Portuguese legislation for the same purpose (Decree-Law No. 113/2013). The rats were housed in $42 \times 27 \times 16 \mathrm{~cm}$ polypropylene cages with a steel lid and had unrestricted access to food (standard commercial chow) and water. A maximum of two rats were kept in a single cage. The same standard house conditions were used throughout the experiment for all the animals.

In the beginning of the study, the seventy-two rats were randomly distributed into two groups. Thirty-six of the rats (group IFS) were continuously exposed to high intensity and very LFN $(2-20 \mathrm{~Hz}$ / $\mathrm{Lp}=120 \mathrm{~dB}$ ), during a period of 1,6 or 12 weeks. The remaining thirty-six rats (group CTL) were used as age-matched controls and were kept in a silent environment. Each group was divided into three subgroups with twelve specimens and sacrificed after 1, 6 and 12 weeks. All rats were euthanized following overnight fast by inhalation of gaseous carbon dioxide.

\subsection{Electroacoustic experiment}

The electroacoustic experiment was described before (Lousinha et al., 2018). In summary, a pseudo-random waveform in the 2 to $20 \mathrm{~Hz}$ decade band was designed with Matlab based on a bandpass-filtered 30$s$ maximum length sequence segment and the resulting acoustic pressure waveform involved an average sound pressure level of $120 \mathrm{~dB}$ with a tolerance of $\pm 3 \mathrm{~dB}$.

\subsection{Histology}

After exsanguination of the sacrificed rats by puncture of the caudal vena cava, hearts were excised, sectioned transversely from the ventricular apex to the atria and routinely processed for light microscopy. The atrial fragment from each heart was selected for the study. Paraffinembedded sections ( $3.5 \mu \mathrm{m}$ thickness) of the atrial tissue samples were made and dyed according to Chromotrope Aniline Blue (CAB) staining 
protocol. For the histological analysis of fibrosis, digitized images were obtained with an optical microscope (Leica ${ }^{\circledR}$ MZ6), equipped with a digital camera (Leica ${ }^{\circledR}$ DF 290HD), under $400 \times$ magnification [Fig. 1]. For each atrium, three random images of equal area containing fibrosis in the absence of any arterial vessel were selected and analyzed using the image $J$ software (National Institutes of Health, Bethesda, MA, USA). For each image, the blue pixel content was measured relative to the total tissue area (the non-staining sections in interstitial spaces were excluded from quantification), using a color deconvolution method (Ruifrok and Johnston, 2001), and the ratio of fibrosis area to atrial cardiomyocytes area was calculated. The mean ratio of the three images was obtained and used for the comparison between the rats. The researchers, including data collectors and data analysts, were blinded to which group the animals belonged to.

\subsection{Immunohistochemistry}

For the immunohistochemistry study, sections adjacent to those employed for histology were used. Specifically, $3.5 \mu \mathrm{m}$ fixed formalin paraffin embedded tissue sections on charged slides were placed in an oven at $60{ }^{\circ} \mathrm{C}$ for $30 \mathrm{~min}$. Sections were deparaffinized, then endogenous peroxidase was blocked with $3 \%$ hydrogen peroxide distilled water for $10 \mathrm{~min}$ at room temperature (RT). After antigen retrieval, the slides were incubated with rabbit polyclonal anti-Cx43 / GJA1 antibody (abcam, ab11370), 1:1000, at $4{ }^{\circ} \mathrm{C}$ overnight. Anti-rabbit Real Envision ${ }^{\circledR}$ HRP Polymer was applied and incubated for $30 \mathrm{~min}$ at RT. Diaminobenzidine chromogen reagent was applied and incubated. The sections were counterstained with Harris Hematoxylin and finally mounted with a coverslip using Entellan Mounting Medium (Merck, Darmstadt, Germany).

For the quantification of $\mathrm{Cx} 43$, a similar method applied for fibrosis was used. For each atrium, three random images of equal area containing Cx43 immunostaining were selected [Fig. 2] and analyzed using the image $J$ software. For each image, a threshold method was used to determine the number of brown pixels corresponding to $\mathrm{Cx} 43$ staining relative to the total tissue area (interstitial spaces were excluded), and the ratio of $\mathrm{Cx} 43$ area to atrial cardiomyocytes area was calculated and averaged for each animal. As before, the researchers were unaware of which group the animals belonged to.

\subsection{Statistical analysis}

Data are presented as mean \pm SD. A two-way ANOVA model was used to fit the data of the two dependent variables, ratio of atrial fibrosis area / cardiomyocytes area and ratio of $\mathrm{Cx} 43$ area / cardiomyocytes area, in the comparison of IFS-exposed animals and group control. A $p$ value $<.05$ was considered statistically significant.

\section{Results}

The main results are presented in Table 1 .

\subsection{Ratio of atrial fibrosis area/cardiomyocytes area}

No interaction between the two independent factors (exposure to IFS and duration of exposure) was observed $(p=.762)$ and no significant effects on atrial interstitial fibrosis due to time were detected $(p=.272)$.

Infrasound-exposed animals showed significantly higher ratio of atrial fibrosis area compared to controls $(p<.001)$, independently of time and with an observed power in excess of 95\% [Fig. 3].

\subsection{Ratio of atrial CX43 area/cardiomyocytes area}

There is no interaction between the two independent factors (exposure / time) on their potential effects on the Cx43 modifications $(p=.751)$. The potential effects of exposure are independent of the potential effects of time.

Exposure to IFS has a significant effect on Cx43 modifications $(p=.009)$, with IFS-exposed rats showing significantly lower values, independently of time and with an observed power of 75.9\% [Fig. 4].

Moreover, Cx43 concentration decrease significantly with time $(p=.001)$, independently of exposure to IFS and with an observed power of $95 \%$.

The weight of the rats at euthanasia was considered as a potential covariate in the model for $\mathrm{Cx} 43$, since the two variables present a modest but significant bivariate correlation $(r=-0.246 ; p=.045)$. However, when included in the model with exposure to IFS and duration of exposure as independent factors, the weight did not present any association with $\mathrm{Cx} 43$ nor did the conclusions stated above for this marker change. Therefore, even after controlling for body weight, the effects of exposure and duration of exposure remain virtually unchanged.

\section{Discussion}

The present study evaluated two specific features of myocardial remodeling in rat heart, interstitial fibrosis and $\mathrm{Cx} 43$ modifications, as a consequence of exposure to IFS $(120 \mathrm{~dB},<20 \mathrm{~Hz})$ and validated our initial hypothesis that exposure to different periods of high-intensity IFS can lead to structural atrial remodeling. These results are consistent with those of past investigations from our group, also in Wistar rats (Oliveira et al., 2005; Oliveira et al., 2012; Oliveira et al., 2013; Antunes et al., 2013a; Lousinha et al., 2015; Antunes et al., 2013b; Antunes et al., 2013c; Antunes et al., 2013d; Lousinha et al., 2018), confirming the abnormal proliferation of connective tissue as the main morphological change induced by LFN exposure. In our study, Cx43
Control

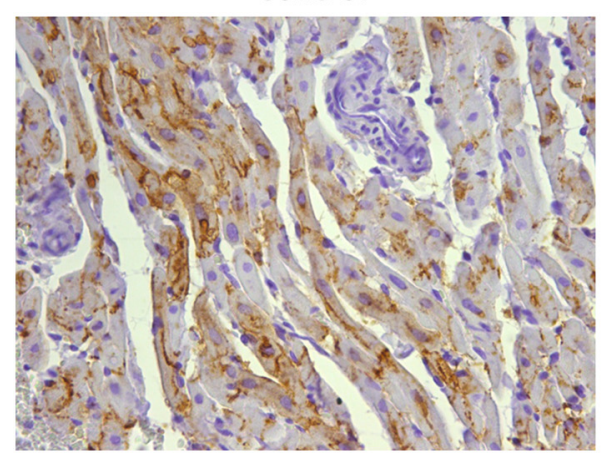

IFS

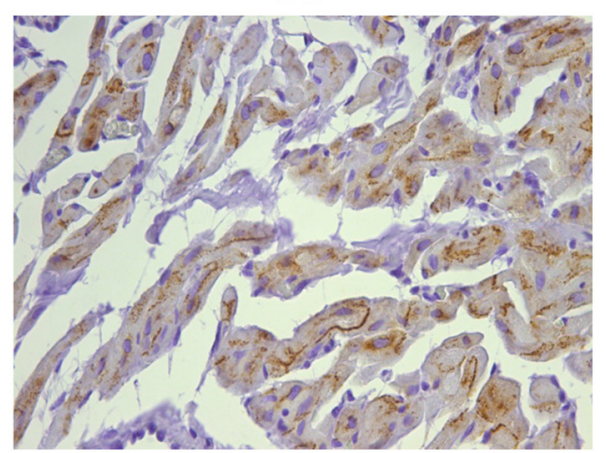

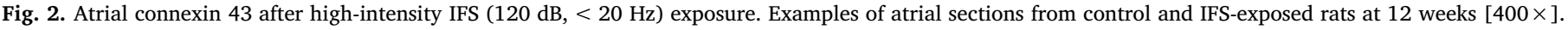
IFS-exposed animals exhibit lower concentrations of Cx43. IFS - Infrasound. 
Table 1

Mean \pm SD of the two measured outcomes in the two groups.

\begin{tabular}{|c|c|c|c|}
\hline & Time of exposure (weeks) & Group IFS $(n=36)$ & Group CTL $(n=36)$ \\
\hline Ratio of atrial fibrosis area / cardiomyocytes area & 1 & $0.0896 \pm 0.04$ & $0.0460 \pm 0.03$ \\
\hline \multirow[t]{2}{*}{ Mean \pm SD } & 6 & $0.0936 \pm 0.03$ & $0.0491 \pm 0.01$ \\
\hline & 12 & $0.1095 \pm 0.04$ & $0.0541 \pm 0.01$ \\
\hline Ratio of atrial CX43 area / cardiomyocytes area & 1 & $0.1100 \pm 0.03$ & $0.1371 \pm 0.03$ \\
\hline \multirow[t]{2}{*}{ Mean \pm SD } & 6 & $0.0829 \pm 0.04$ & $0.1036 \pm 0.03$ \\
\hline & 12 & $0.0834 \pm 0.03$ & $0.0966 \pm 0.03$ \\
\hline
\end{tabular}

IFS - Infrasound; CTL - Control; SD - standard deviation; Cx43 - Connexin 43.

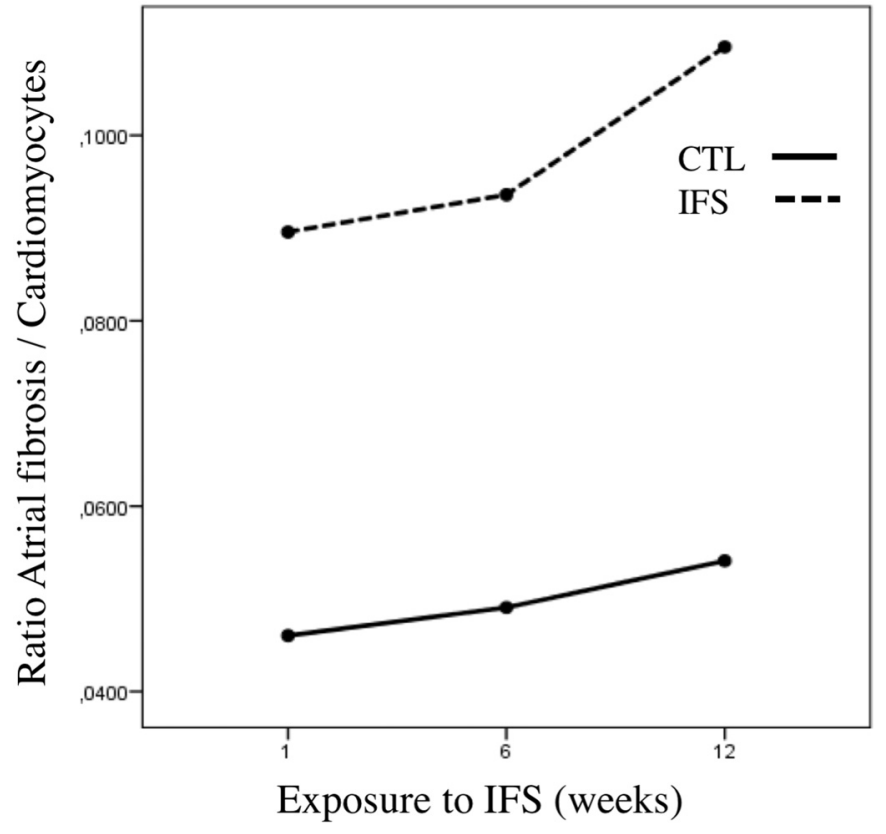

Fig. 3. Ratio of fibrosis area to atrial cardiomyocytes area in IFS-exposed and control animals. The ratio was significantly higher in IFS-exposed animals $(p<.001)$.

also appears to undergo age-dependent loss, as lower concentrations were found among $\geq 22$ weeks-old controls compared to younger rats (16 weeks-old), a result that comes in agreement with previously reported data from Watanabe et al (Watanabe et al., 2004).

High-intensity IFS exposure studies in laboratory animals are scarce but consistently present the deleterious effects of this stressor on the cardiovascular system (Pei et al., 2009; Pei et al., 2007; Pei et al., 2011; Pei et al., 2013). These experiments were conducted with high-pressure IFS (130 dB, $5 \mathrm{~Hz}$ ), in similar conditions to one of our previous studies (Lousinha et al., 2018) and to the present one.

Artificial sources of LFN and IFS include industrial installations and low-speed machinery, like diesel engines and wind turbines (Leventhall, 2007). As previously mentioned, the WHO Regional Office for Europe recognizes that sound with frequencies below $200 \mathrm{~Hz}$ represents an environmental problem (WHO, Regional Office for Europe, 2011; WHO, Regional Office for Europe, 2018). It is widely accepted that noise has impact on public health, although the relative contribute of its intensity, frequency content, mean and peak decibel level, as well as the pattern or duration of the exposure, is not well understood. Lowfrequency noise is implicated in several adverse biological effects in experimental and epidemiological studies (Leventhall, 2004; Leventhall, 2007), which is partially attributed to the characteristics of strong penetration and less attenuation in long distance propagation.

We do not know by which mechanisms noise induces cardiac fibrotic proliferation in rats. The differentiation of cardiac fibroblasts into more active myofibroblasts, a complex and highly regulated process where biochemical and mechanical factors are interdependent, is

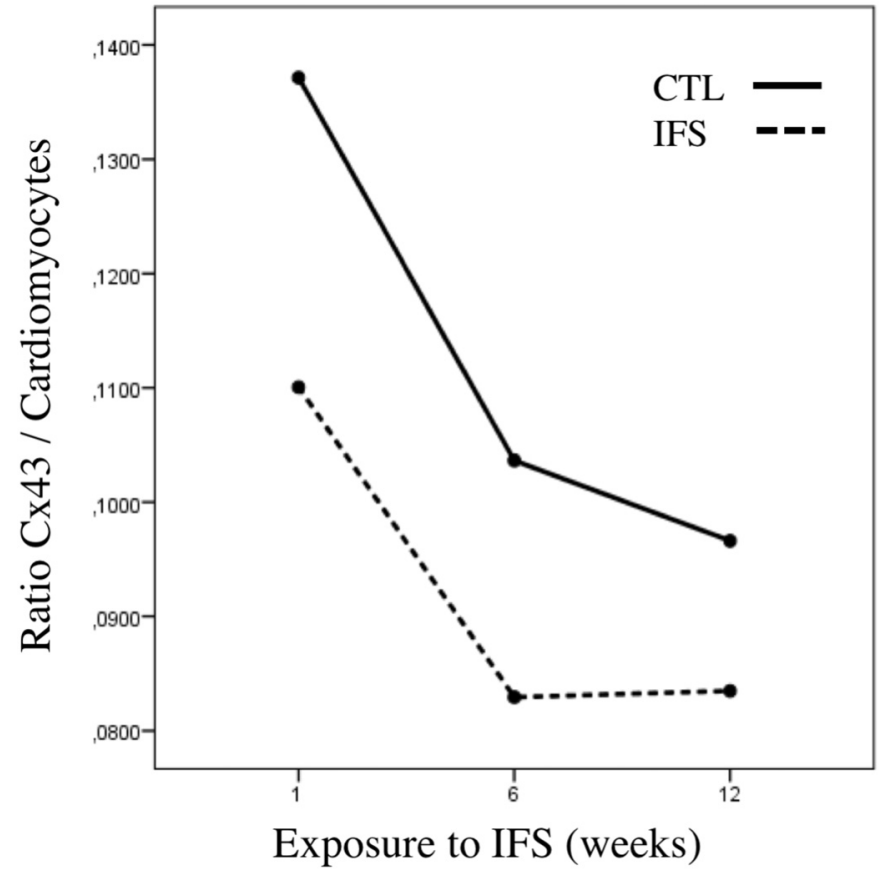

Fig. 4. Ratio of $\mathrm{Cx} 43$ area to atrial cardiomyocytes area in IFS-exposed animals and group control. The ratio was significantly lower in IFS-exposed animals $(p=.009)$. Furthermore, $\mathrm{Cx} 43$ concentration seems to decrease significantly with time $(p=.001)$, independently of exposure and with an observed power of $95 \%$.

the hallmark of cardiac fibrosis (Wynn and Ramalingam, 2012; Yong et al., 2015). Although the role of mechanical factors remains elusive, cardiac fibroblasts exposed to abnormal mechanical conditions such as strain and extracellular matrix stiffness can undergo myofibroblast differentiation, leading to an abnormal accumulation of the extracellular matrix components, such as collagen, in the heart (Dalla-Costa et al., 2010; Hinz, 2010).

High pressure levels of LFN can elicit body vibrations and act as mechanical stressor (Leventhall, 2004; Leventhall, 2007; Randall et al., 1997; Smith, 2002). Humans, as well as other animals, possess inherent specific sound frequencies in certain tissues and organs in the same range of IFS, below $20 \mathrm{~Hz}$ (Leventhall, 2007). Exposure to vibration close to that resonant frequency range can lead to maximum displacement between the organ and the skeletal structure, placing biodynamic strain on the body tissue involved (Randall et al., 1997). We believe this could be among the underlying mechanisms leading to the structural changes we found in our investigations. Comparable to the traditional cardiovascular risk factors, experimental and epidemiological evidence indicates that noise, through auditory and non-auditory effects, may induce activation of different pathways (oxidative stress, inflammation, vascular dysfunction, autonomic imbalance) that ultimately lead to cardiac fibrosis, adverse ventricular and atrial remodeling and arrhythmogenesis (Basner et al., 2014; Cai et al., 2017; Münzel et al., 
2014; Münzel et al., 2017a; Münzel et al., 2017b).

The first study reporting an association between residential exposure to road traffic noise and higher risk for developing $\mathrm{AF}$ was published in 2016, but this association lost statistical significance after adjustment for air pollution (Monrad et al., 2016). Two years later, a group of investigators reported the existence of a significant association between environmental noise exposure, including aircraft and road traffic noise, and AF in a large cohort study, involving more than fourteen thousand participants (Hahad et al., 2018). Furthermore, a third group (Bräuner et al., 2019) found an association between longterm exposure to wind turbine noise, known to generate lower frequencies of sound than road traffic (WHO, Regional Office for Europe, 2018; Seltenrich, 2014), and the risk of incidental AF in a large, nationwide cohort of women above age 44. In these studies, the authors propose the indirect / non-auditory pathway of Babisch's noise and stress-reaction model (Babisch et al., 2013) as the most plausible explanation for their findings but AF is an extraordinarily complex arrhythmia involving several pathophysiological mechanisms (Nattel and Harada, 2014) and there are no studies specifically addressing the pathophysiology of noise-induced AF.

Despite the different underlying mechanisms proposed, the most consistently reported structural change in animals and patients with $\mathrm{AF}$ is atrial fibrosis (Kato et al., 2006; Dzeshka et al., 2015). Among the principal causes of cardiac fibrosis are genetic predisposition, old age, mechanical overload of the heart and myocardial infarction (Rohr, 2012). Cardiac fibrotic remodeling distorts the homogeneous electric substrate and leads to abnormal impulse generation and propagation, representing the most thoroughly investigated mechanism in arrhythmogenesis. But the structural correlate of atrial fibrillation also comprises gap junction remodeling (Kostin et al., 2002; Chimenti et al., 2010; Corradi, 2014). The distribution patterns of connexins are comparable between rat, guinea pig, porcine, bovine and human hearts (van Kempen et al., 1995). In rats, atrial remodeling involving increased fibrosis and altered atrial Cx43 expression consistently lead to higher inducibility of AF (Kato et al., 2006; Kim et al., 2011; Yang et al., 2019), regardless of the pathological condition (diabetes, elevated afterload or obstructive sleep apnea), with overlapping findings in humans (RuckerMartin et al., 2006). From the histopathologic standpoint, it is reasonable to assume that IFS-induced atrial remodeling, with increased fibrosis and decreased $\mathrm{Cx} 43$, shares the same functional relevance. Animal models contribute to our knowledge of arrhythmogenesis. The pathophysiological basis of arrhythmias is not completely understood but different types of arrhythmias share similarities in their basic mechanisms (Clauss et al., 2019).

The main limitation of this study is that we did not address the functional relevance of the atrial remodeling involving fibrosis and Cx43 modifications, leaving open the question of whether it can act as an arrhythmogenic substrate. Fundamental mechanisms can potentially be identified in rats and translated into clinical practice, even considering marked electrophysiological differences in comparison to humans. We consider that this is an initial investigation, as translation of our findings and preclinical studies should be conducted in larger animals and would implicate a different research protocol (Clauss et al., 2019). There are other minor limitations. First, due to the small size and the curvature of the atria of the rats, it was not possible to obtain sections in which all the muscle fibers were oriented in the same plane for histologic and immunohystochemical analysis. Second, experimental noise stress models are scarce and, at the present time, a welldefined morphological cardiac model to study the consequences of IFS exposure does not exist. There is a lack of consensus regarding the cardiac cell composition, including fibroblasts, in mammals, with potential variations between species that also depends on the age (Pinto et al., 2016). Finally, concerning the characteristics of noise, environmental studies mostly use A-weighting method to measure noise and focus on sound pressure level, disregarding frequencies. Low-frequency sounds have higher energy than the sounds at mid and higher frequencies and cannot be correctly evaluated using the conventional A-filters (Ziaran, 2014). Nonetheless, we believe that both sound frequency and intensity are key factors and should be considered in future research.

\section{Conclusions}

High-intensity infrasound exposure triggers atrial structural modifications with increased interstitial fibrosis and decreased $\mathrm{Cx} 43$ in rats. The functional consequences of this findings are not known, reinforcing the need for further research concerning the effects of IFS exposure on the heart.

\section{Funding}

This research did not receive any specific grant from funding agencies in the public, commercial or not for profit sectors.

\section{Ethical approval}

All applicable international, national, and/or institutional guidelines for the care and use of animals were followed.

\section{Declaration of Competing Interest}

None.

\section{References}

Antunes, E., Oliveira, P., Oliveira, M.J., Brito, J., Águas, A., Martins dos Santos, J., 2013a. Histomorphometric evaluation of the coronary artery vessels in rats submitted to industrial noise. Acta Cardiol. 68, 241-245. https://doi.org/10.2143/AC.68.3. 2983423.

Antunes, E., Oliveira, P., Borrecho, G., Oliveira, M.J., Brito, J., Águas, A., dos SJ, M., 2013b. Myocardial fibrosis in rats exposed to low-frequency noise. Acta Cardiol. 68, 285-289. https://doi.org/10.2143/AC.68.3.2983417.

Antunes, E., Borrecho, G., Oliveira, P., Alves de Matos, A.P., Brito, J., Águas, A., Martins dos Santos, J., 2013c. Effects of low-frequency noise on cardiac collagen and cardiomyocyte ultrastructure: an immunohistochemical and electron microscopy study. In: J Clin Exp Pathol. 6. pp. 2333-2341 PMCID:PMC3816801.

Antunes, E., Borrecho, G., Oliveira, P., Brito, J., Águas, A., Martins dos Santos, J., 2013d. Immunohistochemical evaluation of cardiac connexin43 in rats exposed to low-frequency noise. Int. J. Clin. Exp. Pathol. 6, 1874-1879 PMCID:PMC3759495.

Babisch, W., Pershagen, G., Selander, J., Houthuijs, D., Breugelmans, O., Cadum, E., Vigna-Taglianti, F., Katsouyanni, K., Haralabidis, A.S., Dimakopoulou, K., Sourtzi, P., Floud, S., Hansell, A.L., 2013. Noise annoyance - a modifier of the association between noise level and cardiovascular health? Sci. Total Environ. 452, 50-57. https:// doi.org/10.1016/j.scitotenv.2013.02.034.

Basner, M., Babisch, W., Davis, A., Brink, M., Clark, C., Janssen, S., Stansfeld, S., 2014. Auditory and non-auditory effects of noise on health. Lancet 383, 1325-1332. https://doi.org/10.1016/S0140-6736(13)61613-X.

Beranek, L.L., 2006. Basic acoustical quantities: Levels and decibels. In: Vér, I.L., Beranek, L.L. (Eds.), Noise and Vibration Control Engineering. John Wiley and Sons, New Jersey, pp. 1-24.

Bräuner, E.V., Jørgensen, J.T., Duun-Henriksen, A.K., Backalarz, C., Laursen, J.E., Pedersen, T.H., Simonsen, M.K., Andersen, Z.J., 2019. Long-term wind turbine noise exposure and the risk of incident atrial fibrillation in the Danish Nurse cohort. Environ. Int. 130, 104915. https://doi.org/10.1016/j.envint.2019.104915.

Cai, Y., Hansell, A.L., Blangiardo, M., Burton, P.R., BioSHaRE, H.K., Doiron, D., Fortier, I., Gulliver, J., Hveem, K., Mbatchou, S., Morley, D.W., Stolk, R.P., Zijlema, W.L., Elliott, P., Hodgson, S., 2017. Long-term exposure to road traffic noise, ambient air pollution, and cardiovascular risk factors in the HUNT and lifeline cohorts. Eur. Heart J. 38, 2290-2296. https://doi.org/10.1093/eurheartj/ehx263.

Chimenti, C., Russo, M., Carpi, A., Frustaci, A., 2010. Histological substrate of human atrial fibrillation. Biomed. Pharmacother. 64, 1180-1184. https://doi.org/10.1016/j. biopha.2009.09.017.

Clauss, S., Bleyer, C., Schüttler, D., Tomsits, P., Renner, S., Klymiuk, N., Wakili, R., Massberg, S., Wolf, E., Kääb, S., 2019. Animal models of arrhythmia: classic electrophysiology to genetically modified large animals. Nat. Rev. Cardiol. 16, 457-475. https://doi.org/10.1038/s41569-019-0179-0.

Corradi, D., 2014. Atrial fibrillation from the pathologist's perspective. Cardiovasc. Pathol. 23, 71-84. https://doi.org/10.1016/j.carpath.2013.12.001.

Dalla-Costa, A.P., Clemente, C.F., Carvalho, H.F., Carvalheira, J.B., Nadruz, W., Franchini, K.G., 2010. FAK mediates the activation of cardiac fibroblasts induced by mechanical stress through regulation of the mTOR complex. Cardiovasc. Res. 86, 421-431. https://doi.org/10.1093/cvr/cvp416.

Dzeshka, M.S., Lip, G.Y., Snezhitskiy, V., Shantsila, E., 2015. Cardiac fibrosis in patients 
with atrial fibrillation: mechanisms and clinical implications. J. Am. Coll. Cardiol. 66, 943-959. https://doi.org/10.1016/j.jacc.2015.06.1313.

Hahad, O., Beutel, M., Gori, T., Schulz, A., Blettner, M., Pfeiffer, N., Rostock, T., Lackner, K., Sørensen, M., Prochaska, J.H., Wild, P.S., Münzel, T., 2018. Annoyance to different noise sources is associated with atrial fibrillation in the Gutenberg health study. Int. J. Cardiol. 264, 79-84. https://doi.org/10.1016/j.ijcard.2018.03.126.

Hinz, B., 2010. The myofibroblast: paradigm for a mechanically active cell. J. Biomech. 43, 146-155. https://doi.org/10.1016/j.jbiomech.2009.09.020.

Ising, H., Kruppa, B., 2004. Health effects caused by noise: evidence in the literature from the past 25 years. Noise Health 6, 5-13. https://doi.org/10.1016/j.jbiomech.2009. 09.020 .

Kato, T., Yamashita, T., Sekiguchi, A., Sagara, K., Takamura, M., Takata, S., Kaneko, S., Aizawa, T., Fu, L.T., 2006. What are arrhythmogenic substrates in diabetic rat atria? J. Cardiovasc. Electrophysiol. 17, 890-894. https://doi.org/10.1111/j.1540-8167. 2006.00528.x.

Kim, S.J., Choisy, S.C., Barman, P., Zhang, H., Hancox, J.C., Jones, S.A., James, A.F, 2011. Atrial remodeling and the substrate for atrial fibrillation in rat hearts with elevated afterload. Circ. Arrhythm. Electrophysiol. 4, 761-769. https://doi.org/10. 1161/CIRCEP.111.964783.

Kostin, S., Klein, G., Szalay, Z., Hein, S., Bauer, E.P., Schaper, J., 2002. Structural correlate of atrial fibrillation in human patients. Cardiovasc. Res. 54, 361-379. https:// doi.org/10.1016/s0008-6363(02)00273-0.

Leventhall, H.G., 2004. Low frequency noise and annoyance. Noise Health. 6:59-72 PMID:15273024.

Leventhall, G., 2007. What is infrasound? Prog. Biophys. Mol. Biol. 93, 130-137. https:// doi.org/10.1016/j.pbiomolbio.2006.07.006.

Lousinha, A., Antunes, E., Borrecho, G., Oliveira, M.J., Brito, J., dos Santos, J.M., 2015. Histomorphometric evaluation of the small coronary arteries in rats exposed to industrial noise. Int. J. Mol. Sci. 16, 1095-1104. https://doi.org/10.3390/ ijms160510095.

Lousinha, A., Oliveira, M.J.R., Borrecho, G., Brito, J., Oliveira, P., Oliveira de Carvalho, A., Freitas, D., Águas, A.P., Antunes, E., 2018. Infrasound induces coronary perivascular fibrosis in rats. Cardiovasc. Pathol. 37, 39-44. https://doi.org/10.1016/j. carpath.2018.10.004.

Monrad, M., Sajadieh, A., Christensen, J.S., Ketzel, M., Raaschou-Nielsen, O., Tjønneland, A., Overvad, K., Loft, S., Sørensen, M., 2016. Residential exposure to traffic noise and risk of incident atrial fibrillation: a cohort study. Environ. Int. 92, 457-463. https:// doi.org/10.1016/j.envint.2016.04.039.

Münzel, T., Gori, T., Babisch, W., Basner, M., 2014. Cardiovascular effects of environmental noise exposure. Eur. Heart J. 35, 829-836. https://doi.org/10.1093/ eurheartj/ehu030.

Münzel, T., Sørensen, M., Gori, T., Schmidt, F.P., Rao, X., Brook, J., Chen, L.C., Brook, R.D., Rajagopalan, S., 2017a. Environmental stressors and cardio-metabolic disease: part I - epidemiologic evidence supporting a role for noise and air pollution and effects of mitigation strategies. Eur. Heart J. 38, 550-556. https://doi.org/10.1093/ eurheartj/ehw269.

Münzel, T., Sørensen, M., Gori, T., Schmidt, F.P., Rao, X., Brook, J., Chen, L.C., Brook, R.D., Rajagopalan, S., 2017b. Environmental stressors and cardio-metabolic disease: part II - mechanistic insights. Eur. Heart J. 38, 557-564. https://doi.org/10.1093/ eurheartj/ehw294.

Nattel, S., Harada, M., 2014. Atrial remodelling and atrial fibrillation: recent advances and translational perspectives. J. Am. Coll. Cardiol. 63, 2335-2345. https://doi.org/ 10.1016/j.jacc.2014.02.555.

Oliveira, M.J., Pereira, A.S., Ferreira, P.G., Guimarães, L., Freitas, D., Carvalho, A.P., Grande, N.R., Aguas, A.P., 2005. Arrest in ciliated cell expansion on the bronchial lining of adult rats caused by chronic exposure to noise. Environ. Res. 97, 282-286 https://doi.org/10.1016/j.envres.2004.06.006.

Oliveira, M.J., Freitas, D., Carvalho, A.P., Guimarães, L., Pinto, A., Águas, A.P., 2012. Exposure to industrial wideband noise increases connective tissue in the rat liver. Noise Health 14, 227-229. https://doi.org/10.4103/1463-1741.102959.

Oliveira, P., Brito, J., Mendes, J., da Fonseca, J., Águas, A., Martins dos Santos, J., 2013. Effects of large pressure amplitude low frequency noise in the parotid gland perivasculo-ductal connective tissue. Acta Medica Port. 26, 237-242 PMID:23815838.
Pei, Z., Sang, H., Li, R., Xiao, P., He, J., Zhuang, Z., Zhu, M., Chen, J., Ma, H., 2007. Infrasound-induced hemodynamics, ultrastructure, and molecular changes in the rat myocardium. Environ. Toxicol. 22, 169-175. https://doi.org/10.1002/tox.20244.

Pei, Z., Zhuang, Z., Xiao, P., Chen, J., Sang, H., Ren, J., Wu, Z., Yan, G., 2009. Influence of infrasound exposure on the whole L-type calcium currents in rat ventricular myocytes. Cardiovasc. Toxicol. 9, 70-77. https://doi.org/10.1007/s12012-009-9037-3.

Pei, Z.H., Chen, B.Y., Tie, R., Zhang, H.F., Zhao, G., Qu, P., Zhu, X.X., Zhu, M.Z., Yu, J., 2011. Infrasound exposure induces apoptosis of rat cardiac myocytes by regulating the expression of apoptosis-related proteins. Cardiovasc. Toxicol. 11, 341-346. https://doi.org/10.1007/s12012-011-9126-y.

Pei, Z., Meng, R., Zhuang, Z., Zhao, Y., Liu, F., Zhu, M.Z., Li, R., 2013. Cardiac peroxisome proliferator-activated receptor-y expression is modulated by oxidative stress in acutely infrasound-exposed cardiomyocytes. Cardiovasc. Toxicol. 13, 307-315. https://doi.org/10.1007/s12012-013-9211-5.

Pinto, A.R., Ilinykh, A., Ivey, M.J., Kuwabara, J.T., D’Antoni, M.L., Debuque, R., Chandran, A., Wang, L., Arora, K., Rosenthal, N.A., Tallquist, M.D., 2016. Revisiting cardiac cell composition. Circ. Res. 118, 400-409. https://doi.org/10.1161/ CIRCRESAHA.115.307778.

Randall, J.M., Matthews, R.T., Stiles, M.A., 1997. Resonant frequencies of standing humans. Ergonomics 40, 879-886. https://doi.org/10.1080/001401397187711.

Rohr, S., 2012. Arrhythmogenic implications of fibroblast-myocyte interactions. Circ. Arrhythm. Electrophysiol. 5, 442-452. https://doi.org/10.1161/CIRCEP.110. 957647.

Rucker-Martin, C., Milliez, P., Tan, S., Decrouy, X., Recouvreur, M., Vranckx, R., Delcayre, C., Renaud, J.F., Dunia, I., Segretain, D., Hatem, S.N., 2006. Chronic hemodynamic overload of the atria is an important factor for gap junction remodeling in human and rat hearts. Cardiovasc. Res. 72, 69-79. https://doi.org/10.1016/j. cardiores.2006.06.016.

Ruifrok, A.C., Johnston, D.A., 2001. Quantification of histochemical staining by color deconvolution. Anal. Quant. Cytol. Histol. 4, 291-299. 11531144.

Schust, M., 2004. Effects of low frequency noise up to $100 \mathrm{~Hz}$. Noise Health 6, 73-85 PMID:15273025.

Seltenrich, N., 2014. Wind turbines: a different breed of noise? Environ. Health Perspect. 122, A20-A25. https://doi.org/10.1289/ehp.122-A20.

Smith, S.D., 2002. Characterizing the effects of airborne vibration on human body vibration response. Aviat. Space Environ. Med. 73, 36-45. 11817618.

Tannenbaum, J., Bennett, B.T., 2015. Russel and Burch's 3Rs then and now: the need for clarity in definition and purpose. J. Am. Assoc. Lab. Anim. Sci. 54, 120-132 PMCID:PMC4382615.

van Kempen, M.J., ten Velde, I., Wessels, A., Oosthoek, P.W., Gros, D., Jongsma, H.J., Moorman, A.F., Lamers, W.H., 1995. Differential connexin distribution accommodates cardiac function in different species. Microsc. Res. Tech. 31, 420-436. https://doi.org/10.1002/jemt.1070310511.

Watanabe, M., Ichinose, S., Sunamori, M., 2004. Age-related changes in gap junctional protein of the rat heart. Exp. Clin. Cardiol. 9, 130-132 PMCID:PMC2716267.

WHO, Regional Office for Europe, 2011. Burden of Disease from Environmental Noise: Quantification of Healthy Life Years Lost in Europe.

WHO, Regional Office for Europe, 2018. Environmental Noise Guidelines for the European Region.

Wynn, T.A., Ramalingam, T.R., 2012. Mechanisms of fibrosis: therapeutic translation for fibrotic disease. Nat. Med. 18, 1028-1040. https://doi.org/10.1038/nm.2807.

Yang, X., Zhang, L., Liu, H., Shao, Y., Zhang, S., 2019. Cardiac sympathetic denervation suppresses atrial fibrillation and blood pressure in a chronic intermittent hypoxia rat model of obstructive sleep apnea. J. Am. Heart Assoc. 8, e010254. https://doi.org/ 10.1161/JAHA.118.010254.

Yong, K.W., Li, Y., Huang, G., Lu, T.J., Safwani, W.K., Pingguan-Murphy, B., Xu, F., 2015. Mechanoregulation of cardiac myofibroblast differentiation: implications for cardiac fibrosis and therapy. Am. J. Physiol. Heart Circ. Physiol. 309, H532-H542. https:// doi.org/10.1152/ajpheart.00299.2015.

Ziaran, S., 2014. The assessment and evaluation of low-frequency noise near the region of infrasound. Noise Health 16, 10-17. https://doi.org/10.4103/1463-1741.127848. 